\title{
Transit least-squares survey
}

\section{Discovery and validation of an Earth-sized planet in the four-planet system K2-32 near the 1:2:5:7 resonance}

\author{
René Heller ${ }^{1}$, Kai Rodenbeck ${ }^{1,2}$, and Michael Hippke ${ }^{3}$ \\ ${ }^{1}$ Max Planck Institute for Solar System Research, Justus-von-Liebig-Weg 3, 37077 Göttingen, Germany \\ e-mail: heller@mps.mpg.de \\ 2 Institute for Astrophysics, Georg-August-Universität Göttingen , Friedrich-Hund-Platz 1, 37077 Göttingen, Germany \\ e-mail: rodenbeck@mps.mpg.de \\ ${ }^{3}$ Sonneberg Observatory, Sternwartestraße 32, 96515 Sonneberg, Germany \\ e-mail: michael@hippke.org
}

Received 14 February 2019 / Accepted 30 March 2019

\begin{abstract}
We apply for the first time the transit least-squares (TLS) algorithm to search for new transiting exoplanets. TLS has been developed as a successor to the box least-squares (BLS) algorithm, which has served as a standard tool for the detection of periodic transits. In this proof-of-concept paper, we demonstrate that TLS finds small planets that have previously been missed. We show the capabilities of TLS using the K2 EVEREST-detrended light curve of the star K2-32 (EPIC 205071984), which has been known to have three transiting planets. TLS detects these known Neptune-sized planets K2-32 b, d, and c in an iterative search and finds an additional transit signal with a high signal detection efficiency ( $\mathrm{SDE}_{\mathrm{TLS}}$ ) of 26.1 at a period of $4.34882_{-0.00075}^{+0.0069} \mathrm{~d}$. We show that this additional signal remains detectable $\left(\mathrm{SDE}_{\mathrm{TLS}}=13.2\right)$ with TLS in the K2SFF light curve of K2-32, which includes a less optimal detrending of the systematic trends. The signal is below common detection thresholds if searched with BLS in the K2SFF light curve $\left(\mathrm{SDE}_{\mathrm{BLS}}=8.9\right)$, however, as in previous searches. Markov chain Monte Carlo sampling with the emcee software shows that the radius of this candidate is $1.01_{-0.09}^{+0.10} R_{\oplus}$. We analyzed its phase-folded transit light curve using the vespa software and calculated a false-positive probability FPP $=3.1 \times 10^{-3}$. Taking into account the multiplicity boost of the system, we estimate an FPP $<3.1 \times 10^{-4}$, which formally validates K2-32 e as a planet. K2-32 now hosts at least four planets that are very close to a 1:2:5:7 mean motion resonance chain. The offset of the orbital periods of K2-32 e and b from a 1:2 mean motion resonance agrees very well with the sample of transiting multiplanet systems from Kepler, lending further credence to the planetary nature of K2-32 e. We expect that TLS can find many more transits of Earth-sized and even smaller planets in the Kepler and K2 data that have so far remained undetected with algorithms that search for box-like signals.
\end{abstract}

Key words. eclipses - methods: data analysis - planets and satellites: detection - planets and satellites: individual: K2-32 planetary systems - techniques: photometric

\section{Introduction}

The data from the Kepler primary mission (K1; Borucki et al. 2010), which operated from 2009 to 2013, and from the repurposed K2 mission (Howell et al. 2014), which worked from 2014 to 2018, have both been subject to extensive transit searches. Most of their confirmed or validated planets (2338 from K1 and 359 from K2 $)^{1}$ and of the candidates that are yet to be confirmed ( 2423 from $\mathrm{K} 1$ and 536 from K2) have been found using the box least-squares (BLS) transit search algorithm (Kovács et al. 2002) or similar algorithms searching for box-like flux decreases in stellar light curves (Batalha et al. 2013; Vanderburg et al. 2016; Crossfield et al. 2016, 2018; Mayo et al. 2018; Livingston et al. 2018a,b; Yu et al. 2018; van Sluijs \& Van Eylen 2018).

We developed the transit least-squares (TLS) algorithm (Hippke \& Heller 2019) as the successor of BLS in order to be even more sensitive to smaller possibly sub-Earth-sized planets. Instead of searching for box-like flux decreases in the light curve, TLS is based on an analytical transit model with stellar limb darkening (Manduca et al. 1977; Mandel \& Agol 2002).

\footnotetext{
1 https://exoplanetarchive.ipac.caltech.edu/docs/
} counts_detail.html on 28 March 2019.
The signal detection efficiency of TLS is consequently improved compared to BLS, while the false-positive rate is also suppressed (Hippke \& Heller 2019). Here we use TLS to search for so far unknown planets in the K2 data of K2-32 (EPIC 205071984), and we present our first discovery from our new data analysis campaign, the TLS survey.

Three planets have previously been reported around K2-32 by Vanderburg et al. (2016), who formally designated them as candidates, and by Crossfield et al. (2016), who validated them as planets $\mathrm{K} 2-32 \mathrm{~b}$, c, and d using additional high-resolution spectroscopy and independent stellar photometry to feed the statistical vetting software vespa (Morton 2012, 2015). Mayo et al. (2018), also using vespa and additional adaptive optics observations, again detected the three transiting objects and determined their probabilities of being an eclipsing binary, background eclipsing binary, or hierarchical eclipsing binary each to be $<10^{-4}$. Most important, all these previous detections of $\mathrm{K} 2-32 \mathrm{~b}$, c, and $\mathrm{d}$ were achieved in searches for box-like transit signals. Vanderburg et al. (2016) used BLS, Crossfield et al. (2016) used the TERRA software (Petigura et al. 2013a), which includes a search for box-like transit signals very much like BLS, and Mayo et al. (2018) also used BLS. K2-32 b, c, and d have also 


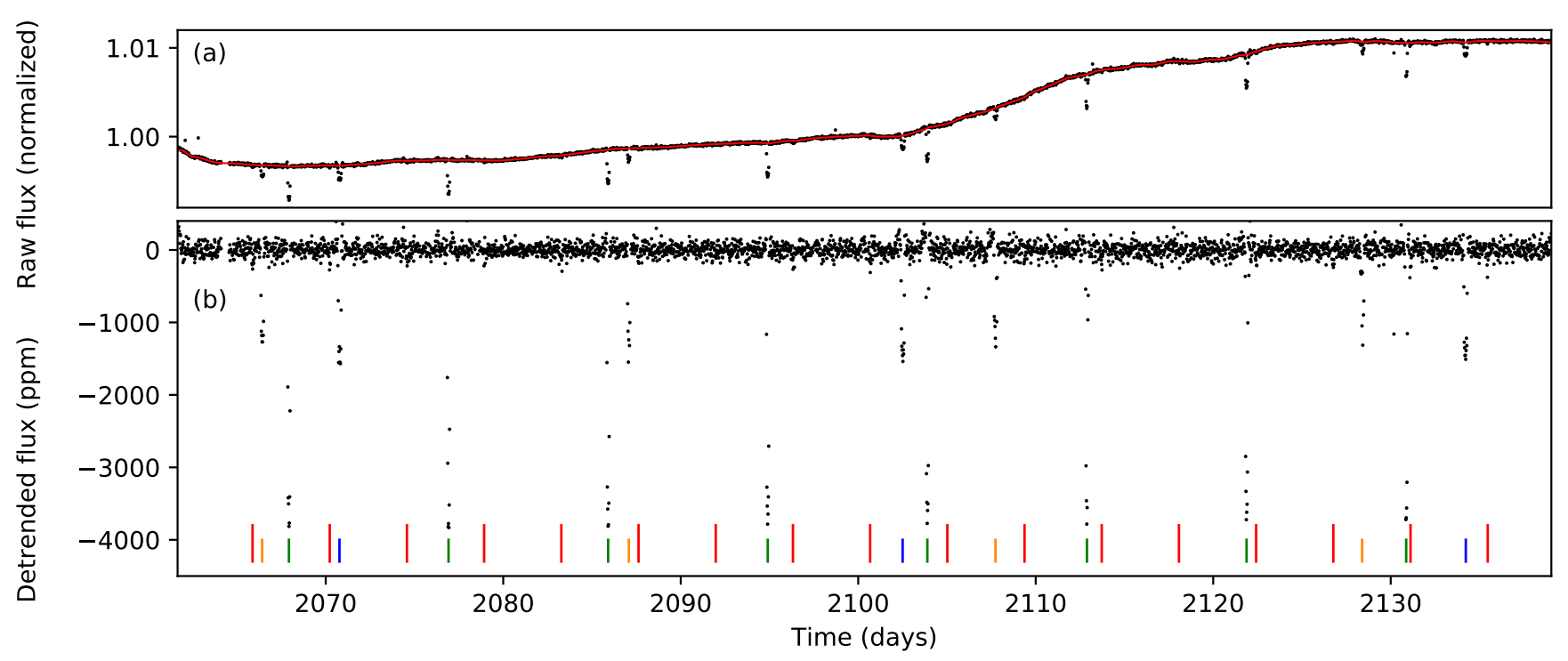

Fig. 1. K2 light curve of K2-32. Panel a: after correction for systematic effects with EVEREST. The red line shows our running median filter. Panel $b$ : detrended light curve obtained by dividing the EVEREST light curve by the running median. Transits detected with TLS are highlighted with green (K2-32 b), magenta (K2-32 c), blue (K2-32 d), and stretched red (K2-32 e) vertical bars.

been confirmed through stellar radial velocity measurements by Dai et al. (2016) and Petigura et al. (2017).

\section{Methods}

\subsection{Target selection}

In this paper, we move on from the testing phase of TLS (Hippke \& Heller 2019) and apply TLS to real light curves. In this initial phase of the TLS survey, we restrict ourselves to multiplanet systems from the Kepler mission because they have been shown to exhibit extremely low false-positive probabilities (FPP; Lissauer et al. 2012). Among the systems that we studied, K2-32 with its three previously known planets stood out with our detection of a highly significant fourth transit signal, the method of which we describe in the following.

\subsection{Transit search}

The light curve of K2-32 from campaign 2 contains $77.4 \mathrm{~d}$ of almost uninterrupted observations with 3527 useful exposures at a cadence of $30 \mathrm{~min}$. We ignored the first 64 exposures in the light curve because they are affected by strong systematics. We removed outliers, defined as data points $>3 \sigma$ above the running mean, from the publicly available ${ }^{2} \mathrm{~K} 2$ light curve of K2-32, which has been corrected for instrumental effects with EVEREST (Luger et al. 2016, 2018) (Fig. 1a). We then removed stellar variability and other trends using a median filter with a window size of $1 \mathrm{~d}$. The resulting detrended light curve is shown in Fig. $1 b$.

We tested other window sizes and found that a width of $1 \mathrm{~d}$ offers the best compromise between both sufficient removal of unwanted variability and preservation of transit signals with durations of up to several hours. Kepler's third law of motion (Kepler 1619) predicts that planets with orbital periods $<80 \mathrm{~d}$ around sun-like stars have transit durations shorter than about $8 \mathrm{~h}$ (see Fig. 5 in Hippke \& Heller 2019). For K2-32 in particular, the

\footnotetext{
2 https://archive.stsci . edu/hlsps/everest/v2/c02/ 205000000/71984/hlsp_everest_k2_1lc_205071984-c02_ kepler_v2.0_lc.fits
}

maximum duration of a planet with a single transit in the $80 \mathrm{~d} \mathrm{~K} 2$ light curve on a circular orbit is $5.7 \mathrm{~h}$, and for a planet to exhibit two transits (hence $P<40 \mathrm{~d}$ ), the maximum transit duration is $4.8 \mathrm{~h}$, suggesting that a $1 \mathrm{~d}$ width for our median filter hardly affects physically plausible transit signals.

We applied the publicly available ${ }^{3}$ python implementation of TLS (Hippke \& Heller 2019) using the stellar limb-darkening, mass, and radius estimates available from the EPIC catalog (Huber et al. 2016). We used TLS version 1.0.16 in its default parameterization, but set the maximum trial period equal to the length of the light curve in order to search for possible single transits.

\subsection{Markov chain Monte Carlo analysis of the transits}

To refine the planetary parameters we used the Markov chain Monte Carlo (MCMC) sampler emcee (Foreman-Mackey et al. 2013). As inputs to emcee, we provided the times of the midpoints of the first transit $\left(T_{0}\right)$ and the orbital period $(P)$ obtained with TLS for each planet. $P, T_{0}$, the planet-to-star radius ratio $\left(R_{\mathrm{p}} / R_{\mathrm{S}}\right)$, and the transit impact parameter $(b)$ served as model parameters for each planet, while the stellar density $\left(\rho_{\mathrm{s}}\right)$ and two limb-darkening coefficients for a quadratic limb darkening law (Kipping 2013) were global parameters for all transits. We ran the MCMC analysis with 100 walkers, with each walker performing 200000 steps. The first half of each walk was discarded to ensure that we preserved only burned-in MCMC chains.

\section{Results}

\subsection{Transit detection and characterization}

In Fig. 2 we show the signal detection efficiency ( $\mathrm{SDE}_{\mathrm{TLS}}$ ) periodograms of our iterative transit search with TLS in the K2 light curve that has been extracted with EVEREST. Each iteration ignores the in-transit data corresponding to transits detected in previous iterations. TLS first found planet $b$ (top panel) because it is both the largest planet and exhibits more transits than planets $\mathrm{c}$ and $\mathrm{d}$ in the light curve. Next, TLS found planet $\mathrm{d}$ (second

\footnotetext{
3 https://github.com/hippke/tls
} 
R. Heller et al.: Transit least-squares survey. I.

Table 1. Characterization of the new planet K2-32 e from MCMC model fitting to the full set of transits in the EVEREST light curve of K2-32.

\begin{tabular}{|c|c|c|c|c|c|c|c|c|}
\hline & \multicolumn{2}{|c|}{ Planet e } & \multicolumn{2}{|c|}{ Planet b } & \multicolumn{2}{|c|}{ Planet c } & \multicolumn{2}{|c|}{ Planet d } \\
\hline & $\mathrm{ML}^{(a)}$ & Median $_{\text {-err. }}^{+ \text {err. }}$ & $\mathrm{ML}^{(a)}$ & Median $_{\text {-err. }}^{\text {err. }}$ & $\mathrm{ML}^{(a)}$ & Median $_{- \text {err. }}^{\text {+err. }}$ & $\mathrm{ML}^{(a)}$ & Median $_{- \text {err. }}^{+ \text {err. }}$ \\
\hline$P(\mathrm{~d})$ & 4.34911 & $4.34882_{-0.00075}^{+0.00069}$ & 8.991866 & $8.991828_{-0.000084}^{+0.000083}$ & 20.66157 & $20.66186_{-0.00098}^{+0.00102}$ & 31.7145 & $31.7142_{-0.0010}^{+0.0011}$ \\
\hline$T_{0}(\mathrm{~d})^{(b)}$ & 0.8799 & $0.8860_{-0.0079}^{+0.0085}$ & 2.92698 & $2.92713_{-0.00034}^{+0.00035}$ & 1.4231 & $1.4227_{-0.0021}^{+0.0021}$ & 5.7923 & $5.7913_{-0.0017}^{+0.0014}$ \\
\hline$R_{\mathrm{p}} / R_{\mathrm{s}}$ & 0.01080 & $0.01090_{-0.00051}^{+0.00052}$ & 0.05347 & $0.05382_{-0.00068}^{+0.00076}$ & 0.029430 & $0.029664_{-0.00053}^{+0.00058}$ & 0.03534 & $0.03551_{-0.00062}^{+0.00064}$ \\
\hline$b$ & 0.029 & $0.22_{-0.15}^{+0.17}$ & 0.047 & $0.15_{-0.10}^{+0.16}$ & 0.24 & $0.28_{-0.08}^{+0.10}$ & 0.451 & $0.463_{-0.030}^{+0.057}$ \\
\hline
\end{tabular}

Notes. ${ }^{(a)} \mathrm{ML}=$ value with maximum likelihood. ${ }^{(b)} T_{0}=$ BKJD $-2065 \mathrm{~d}$ with the Barycentric Kepler Julian Day BKJD $=$ BJD $-2454833.0 \mathrm{~d}$.

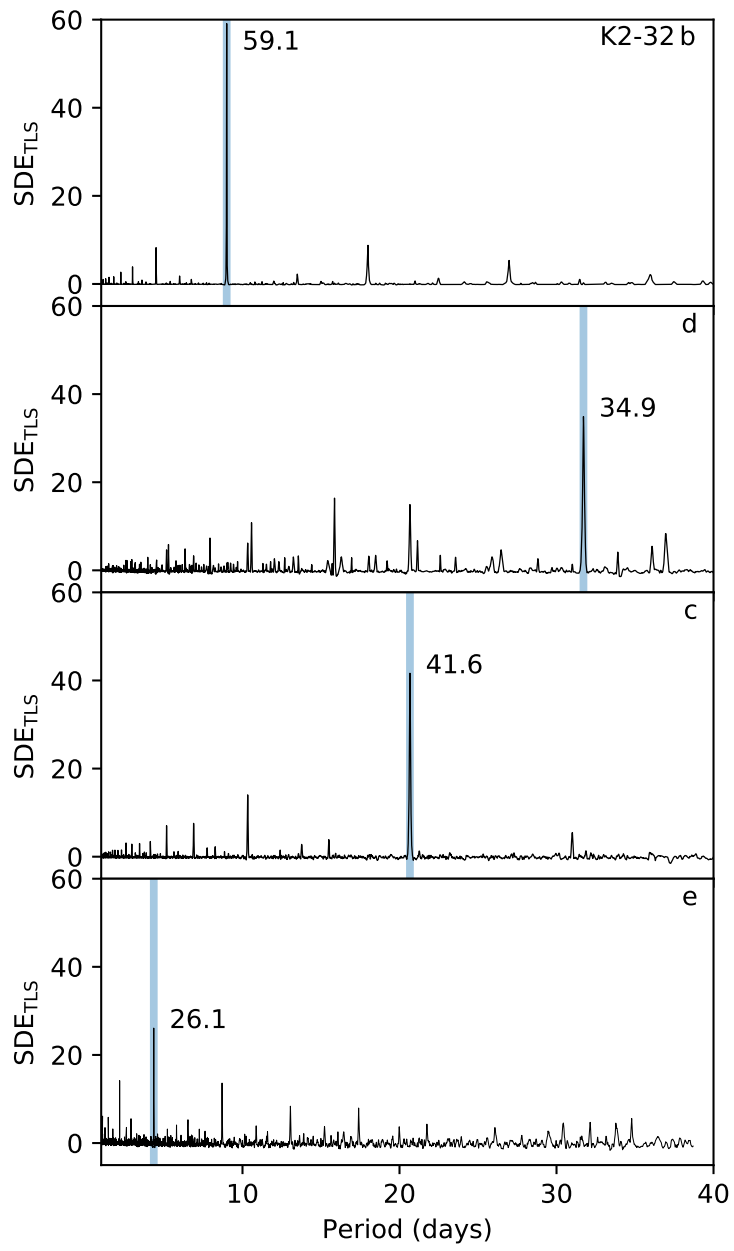

Fig. 2. Signal detection efficiencies of our successive transit search after masking out previously detected transits.

panel), which is the second largest planet in the system. Planet c was detected in our third iteration with TLS (third panel). In a fourth run then, we found another strong peak $\left(\mathrm{SDE}_{\mathrm{TLS}}=26.1\right)$ at a period of $4.34882_{-0.00075}^{+0.0006} \mathrm{~d}$, which we preliminary referred to as a candidate dubbed K2-32 e. We also executed a fifth search after masking out all transits from the four planets, but we did not find any other significant signals.

Figure 3 illustrates the phase-folded light curves of K2-32 e (top panel) and of the previously discovered planets $\mathrm{b}, \mathrm{c}$, and $\mathrm{d}$ from top to bottom. The shallow transit depth of about 200 parts per million (ppm) is immediately suggestive of an Earthsized planet, given that K2-32 is a somewhat subsolar-sized

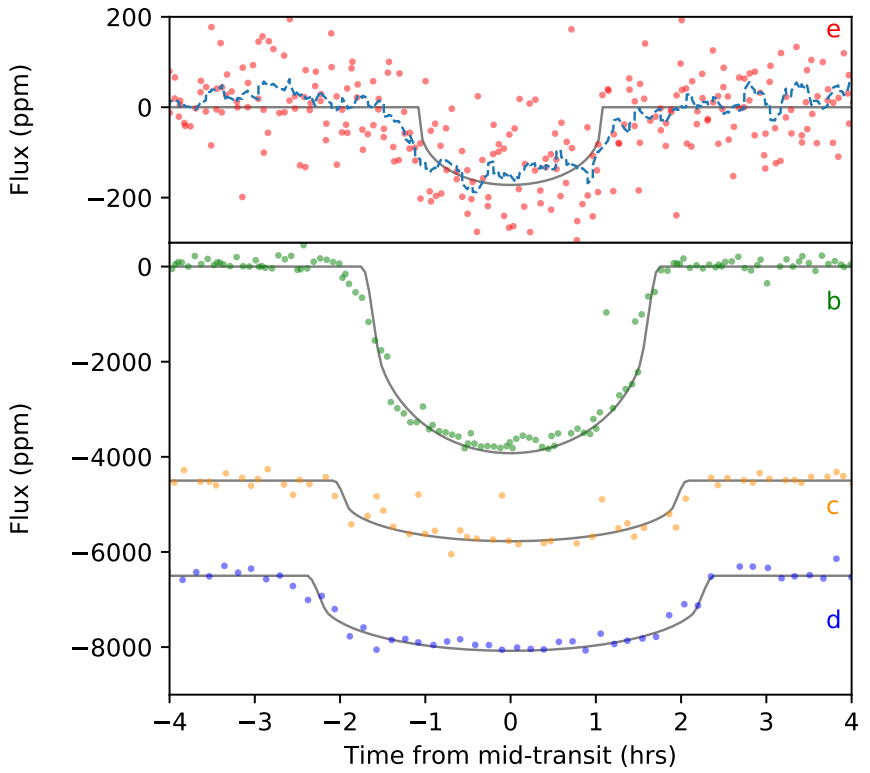

Fig. 3. Phase-folded transit light curves of all planets. Dots show the K2 data, lines represent our best-fit MCMC models. The ordinates in the top and bottom panels have different scales. Top panel: transit dip of $\mathrm{K} 2-32 \mathrm{e}$ is shown together with a sliding mean (dashed line) of 11 cadences in width.

$\mathrm{K}$ dwarf star. We note that the ordinate in the top panel, which shows the transit data of the new object, is an 18-fold zoom compared to the bottom panel, which contains the data of planets b, c, and d.

To further characterize the new planet candidate, we applied the MCMC sampler to the entire light curve. The stellar density was found to be $1.42_{-0.15}^{+0.08}$ times the solar density and the best-fit limb-darkening parameters are $q_{1}=0.57_{-0.18}^{+0.23}$ and $q_{2}=0.47_{-0.11}^{+0.16}$. Our results for K2-32 e are shown in Table 1 , and our results from the MCMC sampling for the previously known planets $\mathrm{K} 2-32 \mathrm{~b}, \mathrm{c}$, and d are compared to the literature values in Table 2.

Figure 4 is a visualization of the physical and orbital characteristics. K2-32 is indicated as a cropped orange shaded circle, and its planets are denoted by empty circles with radii to scale with the star. The vertical bars denote the uncertainties in $b$, and the transit curves are to-scale representations of the best-fit transit models shown in Fig. 3. Orbital resonances are indicated at the bottom of this chart, with the 1:1 resonance referring to the innermost planet, K2-32 e. Interestingly, we found that this four-planet system is close to being in a 1:2:5:7 mean motion resonance (MMR) chain. At the same time, however, the system 
Table 2. System parameterization of K2-32 and its planets and comparison to literature values.

\begin{tabular}{|c|c|c|c|c|c|c|}
\hline & Star & Planet e & Planet $b$ & Planet c & Planet d & Ref. \\
\hline \multirow[t]{5}{*}{$P(\mathrm{~d})$} & & & 8.99213 & 20.6602 & 31.7154 & C16 \\
\hline & & & $8.99218_{-0.00020}^{+0.00020}$ & $20.65614_{-0.00598}^{+0.00598}$ & $31.71922_{-0.00236}^{+0.00236}$ & D16 \\
\hline & & & $8.99213_{-0.00016}^{+0.00016}$ & $20.6602_{-0.0017}^{+0.0017}$ & $31.7154_{-0.0022}^{+0.0022}$ & P17 \\
\hline & & & $8.99194_{-0.00016}^{+0.00016}$ & $20.6616_{-0.0018}^{+0.0017}$ & $31.7151_{-0.0026}^{+0.0022}$ & M18 \\
\hline & & $4.34882_{-0.00075}^{+0.00069}$ & $8.991828_{-0.000084}^{+0.000083}$ & $20.66186_{-0.00098}^{+0.00102}$ & $31.7142_{-0.0010}^{+0.0011}$ & H19 \\
\hline \multirow[t]{5}{*}{$R$} & & & $5.62 R_{\oplus}$ & $3.32 R_{\oplus}$ & $3.77 R_{\oplus}$ & $\mathrm{C} 16^{(a)}$ \\
\hline & $0.87_{-0.05}^{+0.05} R_{\odot}$ & & $5.38_{-0.35}^{+0.35} R_{\oplus}$ & $3.48_{-0.42}^{+0.97} R_{\oplus}$ & $3.75_{-0.40}^{+0.40} R_{\oplus}$ & D16 \\
\hline & $0.845_{-0.035}^{+0.044} R_{\odot}$ & & $5.13_{-0.28}^{+0.28} R_{\oplus}$ & $3.01_{-0.25}^{+0.25} R_{\oplus}$ & $3.43_{-0.35}^{+0.35} R_{\oplus}$ & $\mathrm{P} 17$ \\
\hline & $0.839_{-0.026}^{+0.021} R_{\odot}$ & & $5.17_{-0.20}^{+0.16} R_{\oplus}$ & $3.12_{-0.18}^{+0.12} R_{\oplus}$ & $3.41_{-0.26}^{+0.14} R_{\oplus}$ & M18 \\
\hline & & $1.01_{-0.09}^{+0.10} R_{\oplus}$ & $4.96_{-0.27}^{+0.33} R_{\oplus}$ & $2.74_{-0.16}^{+0.10} R_{\oplus}$ & $3.27_{-0.19}^{-0.23} R_{\oplus}$ & $\mathrm{H} 19^{(b)}$ \\
\hline \multirow[t]{2}{*}{$M$} & $0.87_{-0.04}^{+0.04} M_{\odot}$ & & $21.1_{-5.9}^{+5.9} M_{\oplus}$ & $<8.1 M_{\oplus}(95 \%$ conf. $)$ & $<35.0 M_{\oplus}(95 \%$ conf. $)$ & D16 \\
\hline & $0.856_{-0.028}^{+0.028} M_{\odot}$ & & $16.5_{-2.7}^{+2.7} M_{\oplus}$ & $<12.1 M_{\oplus}(95 \%$ conf. $)$ & $10.3_{-4.7}^{+4.7} M_{\oplus}$ & $\mathrm{P} 17$ \\
\hline \multirow[t]{3}{*}{$a(\mathrm{AU})$} & & & 0.0811 & 0.14120 & 0.1879 & $\mathrm{C} 16^{(a)}$ \\
\hline & & & $0.08036_{-0.00088}^{+0.00088}$ & $0.1399_{-0.0015}^{+0.0015}$ & $0.1862_{-0.0020}^{+0.0020}$ & P17 \\
\hline & & $0.04951_{-0.00055}^{+0.00055}$ & $0.08035_{-0.00089}^{+0.00089}$ & $0.1399_{-0.0016}^{+0.0016}$ & $0.1862_{-0.0021}^{+0.0021}$ & $\mathrm{H} 19^{(c)}$ \\
\hline \multirow[t]{4}{*}{$\operatorname{FPP}^{(d)}$} & & & 0 & $5 \times 10^{-4}$ & $5.4 \times 10^{-6}$ & $\mathrm{C} 16^{(a)}$ \\
\hline & & & $<1 \times 10^{-3}$ & $2.2 \times 10^{-2}$ & $<1 \times 10^{-3}$ & S16 \\
\hline & & & $<1 \times 10^{-4}$ & $<1 \times 10^{-4}$ & $<1 \times 10^{-4}$ & M18 \\
\hline & & $3.1 \times 10^{-3}$ & $5.2 \times 10^{-3}$ & $4.9 \times 10^{-3}$ & $7.2 \times 10^{-3}$ & H19 \\
\hline
\end{tabular}

Notes. ${ }^{(a)}$ Based on stellar classification $\left(0.92 \pm 0.07 R_{\odot}, 0.88 \pm 0.03 M_{\odot}\right)$ by Huber et al. $(2016) .{ }^{(b)}$ Planetary radii and $68.3 \%$ confidence intervals based on our measured planet-to-star radius ratio and stellar radius estimates from P17. ${ }^{(c)}$ Semimajor axes and $68.3 \%$ confidence intervals based on our measurements of the orbital periods and stellar mass estimates from P17. ${ }^{(d)} \mathrm{FPP}=$ false-positive probability. Values do not contain the "multiplicity boost" (reduction in FPP) but are detailed in S16.

References. C16: Crossfield et al. (2016); D16: Dai et al. (2016); S16: Sinukoff et al. (2016); P17: Petigura et al. (2017); M18: Mayo et al. (2018); H19: this study.
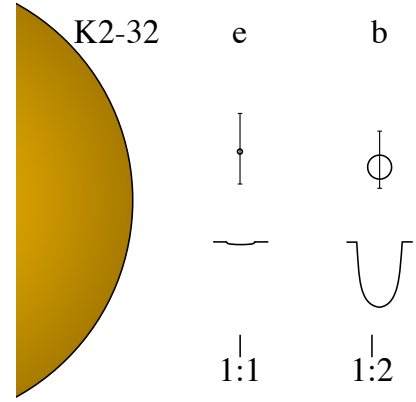

b

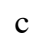

d

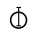

$\Phi$

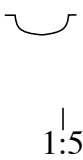

$1: 5$

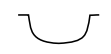

$1: 7$
Fig. 4. System architecture. Stellar and planetary radii are to scale. Planetary distances to the star are mutually to scale, but not with respect to the radii. The shapes of the transit light curves are to scale as well. Orbital resonances are indicated with respect to the innermost planet K2-32 e. The error bars denote our uncertainties in the transit impact parameter (Table 1).

is clearly out of this resonance compared to our measurement uncertainties.

\subsection{False-positive vetting and validation}

We used the publicly available vespa software (Morton 2012, 2015) to evaluate the FPP of K2-32 e. In brief, vespa takes the phase-folded transit light curve together with the celestial coordinates and stellar parameters to calculate the probabilities of the data being caused by non-associated blended eclipsing binaries, hierarchical triples, genuine eclipsing binaries, and non-associated stars with transiting planets. We supplied vespa with the celestial coordinates of $\mathrm{K} 2-32, P$ and $R_{\mathrm{p}} / R_{\mathrm{s}}$ of our candidate signal (Table 1), the phase-folded transit light curve (Fig 3, top panel), the stellar parameters as determined spectroscopically by Petigura et al. (2017) (Table 2), and the 2MASS broadband photometry $(J=10.404 \pm 0.024, H=9.993 \pm 0.025$, $K=9.821 \pm 0.019$; Cutri et al. 2003) following the "Lessons Learned" section in Petigura et al. (2017). As a limiting aperture within which the transits are observed, we referred to adaptive optics and K2 light curve analyses of Sinukoff et al. (2016), who found that the transits are localized within 8 arcseconds of K2-32. vespa returned an FPP of $3.1 \times 10^{-3}$ for K2-32 e.

Although this number formally validates K2-32 e as a planet already (the commonly used FFP threshold for validation is $1 \%$ ), it still does not consider the "multiplicity boost" (reduction in FPP) inherent to planet candidates in multiplanet systems. Planet candidates in systems known to already harbor planets have a much lower FPP than single candidates (Lissauer et al. 2012). We used the values of the homogeneous K2 exoplanet survey by Vanderburg et al. (2016) with $n_{\mathrm{t}}=59,174$ as the number of target stars (K2 campaigns 0 to 3 ), $n_{\mathrm{c}}=234$ as the number of $\mathrm{K} 2$ planet candidates, and $n_{\mathrm{m}}=26$ as the number of candidate multiplanet systems and plugged them into Eq. (6) of Lissauer et al. (2012). We found that the total number (the expectation value) of K2 systems like K2-32 with two or more known planets 
and one additional false positive ranges between 0.01 , assuming a true-positive rate (or planet fidelity) of $90 \%$, and 0.05 , assuming a true-positive rate of $50 \%$. These estimates increased our confidence that $\mathrm{K} 2-32 \mathrm{e}$ is a true planet because both values are much lower than 1. Sinukoff et al. (2016) showed that based on the statistical framework of Lissauer et al. (2012), the multiplicity boost is at least an order of magnitude in FPP. As a consequence, a conservative estimate of the FPP for K2-32 e including the multiplicity boost is $<3.1 \times 10^{-4}$.

With the depth of the secondary transit $\left(\delta_{2}\right)$ being of the order of $\left(R_{\mathrm{p}} / a\right)^{2}$, or $0.056 \mathrm{ppm}$ in the case of $\mathrm{K} 2-32 \mathrm{e}$, we expect this phenomenon to be invisible to $\mathrm{K} 2$. Thus, as an additional test for a false positive, for example, caused by an eclipsing binary, we measured $\delta_{2}$ in that part of the light curve where the secondary transit can be expected on a circular orbit. We detected a $8 \pm 8 \mathrm{ppm}$ dip, that is to say, a $1 \sigma$ signal that is statistically compatible with noise.

\subsection{Comparison of the BLS and TLS performances}

One of the most pressing questions is why K2-32 e has not been detected in previous transit searches, for instance, those by Vanderburg et al. (2016) and Crossfield et al. (2016).

For our comparison of the transit search results obtained with BLS and TLS, we have to keep in mind that the definitions of the signal detection efficiency introduced by Kovács et al. (2002) for BLS (SDE BLS $_{\text {) and by Hippke \& Heller (2019) for TLS (SDE }}$ TLS) are different ${ }^{4}$. While $\mathrm{SDE}_{\mathrm{BLS}}$ is more closely related to the mean transit depth, $\mathrm{SDE}_{\mathrm{TLS}}$ is derived from a $\chi^{2}$ statistic and therefore naturally tends to produce higher values for a given transit-like signal. This principal difference between $\mathrm{SDE}_{\mathrm{BLS}}$ and $\mathrm{SDE}_{\mathrm{TLS}}$ must also be noted in the context of the heuristically chosen detection thresholds of typically between 8 and 9 in other studies. We have shown in Hippke \& Heller (2019), using injected transits of Earth-like planets in the light curves of Sun-like stars with white noise, that a false-positive rate of $1 \%$ is achieved at virtually the same signal detection efficiency value of 7 for both BLS (here $\mathrm{SDE}_{\mathrm{BLS}}=7$ ) and TLS (here $\mathrm{SDE}_{\mathrm{TLS}}=7$ ). Although the statistics of BLS and TLS are different, they produced the same SDE values for this particular set of simulated data. Most important, however, the true-positive rates were $\sim 76 \%$ for BLS and $\sim 93 \%$ for TLS. The TERRA algorithm uses yet another search statistic, the signal-to-noise ratio $(\mathrm{S} / \mathrm{N})$, with a detection threshold set to a nominal value of 12 "by trial and error" (Petigura et al. 2013b).

Vanderburg et al. (2016) and Crossfield et al. (2016) both used the K2 light curve produced with the "K2 self-flat-fielding" (K2SFF) pipeline, which removes most of the instrumental jitter (Vanderburg \& Johnson 2014). This light curve contains a somewhat stronger noise than the light curve extracted with the EVEREST pipeline, and we therefore need to examine whether our discovery of $\mathrm{K} 2-32 \mathrm{e}$ is owing to improvements of the $\mathrm{K} 2$ light curve extraction and systematic detrending or due to the use of TLS instead of BLS. We thus repeated our analysis using the K2SFF data and tested both TLS and BLS, the results of which are shown in Fig. 5.

In the left column of Fig. 5, we present an iterative transit search in the K2SFF light of K2-32 with BLS. We successively detected planets $b, d$, and $c$ with very strong $S_{D E} E_{B L S}$ signals (see labels), whereas planet e produced an $\mathrm{SDE}_{\mathrm{BLS}}$ of just 8.9 in the fourth iteration, which is below the widely used detection

4 See Eqs. (5) and (6) in Kovács et al. 2002 and Eqs. (3) and (5) in Hippke \& Heller 2019, respectively. threshold value of 9 (Vanderburg et al. 2016; Crossfield et al. 2016). We verified that the addition of a smoothing filter to the $\mathrm{SDE}_{\mathrm{BLS}}$ spectrum, similar to what is done in TLS to the $\mathrm{SDE}_{\mathrm{TLS}}$ spectrum, does not lift this signal above the detection threshold. None of the previous K2 transit surveys by Vanderburg et al. (2016), Crossfield et al. (2016), Petigura et al. (2018), and Mayo et al. (2018) applied such a smoothing filter to their $\mathrm{SDE}_{\mathrm{BLS}}$ or $\mathrm{S} / \mathrm{N}$ spectra.

Moreover, the signature of $\mathrm{K} 2-32 \mathrm{e}$ is only the second strongest signal, while the strongest signal near $P=0.4 \mathrm{~d}$ with an $\mathrm{SDE}_{\mathrm{BLS}}$ of 10.5 is a false positive, as we verified. We found that this false-positive signal is produced by a combination of two characteristics of BLS: the quantification in transit durations in units of cadences and the linear period grid typically used in BLS applications. For example, Petigura et al. (2013b) used "transit durations that are integer numbers of long cadence measurements (...)" with a grid of, for example, " $\Delta T=$ $[3,5,7,10,14,18]$ long cadence measurements". Aliasing effects of duration and period integer multiples cause additional jitter in the BLS spectrum, an effect that is absent in TLS because it does not apply any binning. In addition, the period grid in TLS is not linear (Ofir 2014) because the frequency grid is linear, which intrinsically suppresses the possibility of aliasing. We are therefore able to reproduce the results of previous searches with the traditional BLS algorithm and confirm that K2-32 e remains undetected in the K2SFF light curve.

In the right column of Fig. 5, we show the $\mathrm{SDE}_{\mathrm{TLS}}$ results for an iterative transit search with TLS in the K2SFF data. We found planets $\mathrm{b}, \mathrm{d}$, and $\mathrm{c}$ with $\mathrm{SDE}_{\mathrm{TLS}}$ values comparable to those obtained with BLS, but most interestingly, planet e also passed the detection limit with a significant $S_{D E}$ TLS of 13.2. This is a fascinating real-world example of the simulation-based findings by Hippke \& Heller (2019): the strongest improvement in using TLS instead of BLS occurs for shallow transits or, in this case, for Earth-sized planets.

We also found that planet e can be recovered in the EVEREST data with both BLS $\left(\mathrm{SDE}_{\mathrm{BLS}}=21.3\right)$ and TLS $\left(\mathrm{SDE}_{\mathrm{TLS}}=26.1\right)$. We conclude that if either the TLS search algorithm or the EVEREST data reduction pipeline would have been available to Vanderburg et al. (2016) and Crossfield et al. (2016), they could have found K2-32 e. Most important, however, the combination of EVEREST and TLS leverages the optimal detrending and signal detection procedures.

We also examined the different methods that were used to remove longer-term stellar trends. Vanderburg et al. (2016) and Crossfield et al. (2016) both used spline fits, while we used a running median by default. When we used a spline fit instead of a running median, the $\mathrm{SDE}_{\mathrm{TLS}}$ values obtained with TLS were virtually identical with variations of about 0.1 .

\section{Discussion}

\subsection{K2-32 system}

For now, the mass of K2-32 e remains unconstrained. With one Earth radius in size, a first guess for the mass of $\mathrm{K} 2-32 \mathrm{e}$ is about one Earth mass. Although the densities of planets b, c, and d range between those of Saturn and Neptune, which suggests large and massive gaseous envelopes, it can safely be assumed that K232 e does not carry large amounts of gas. If K2-32 e consisted mostly of gas, its low density would imply a sub-Earth-mass planet. Such a very low-mass (and very low-gravity) planet, however, could hardly hold on to a significant gas envelope, in particular under the effects of extreme stellar irradiation. Assuming 

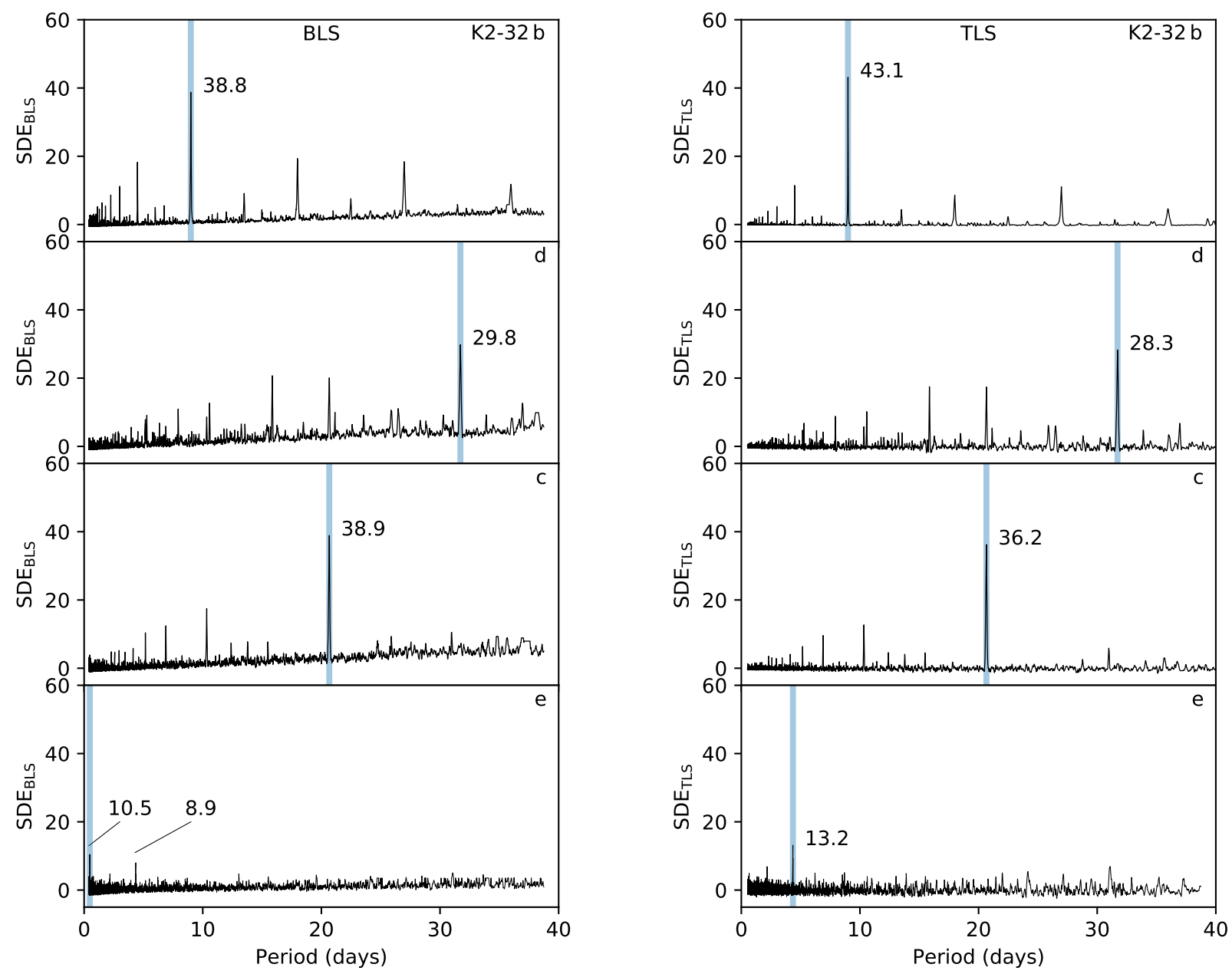

Fig. 5. Iterative transit search using K2SFF data with BLS (left) and TLS (right). Planets K2-32 b, d, and c are successively detected with strong signals (see labels) with both BLS and TLS. Using a common detection threshold value of 9, however, planet e is not detected with BLS $\left(\mathrm{SDE}_{\mathrm{BLS}}=8.9\right)$, but it is detected with TLS $\left(\mathrm{SDE}_{\mathrm{TLS}}=13.2\right)$. The major peak near $P=0.4 \mathrm{~d}$ with $\left(\mathrm{SDE}_{\mathrm{BLS}}=10.5\right)$ in the fourth iteration of $\mathrm{BLS}$ is a false positive (blue marker). It overshadows the true transit signal near $P=4.3 \mathrm{~d}$ with $\left(\mathrm{SDE}_{\mathrm{BLS}}=8.9\right)$.

an Earth-mass $\left(M_{\oplus}\right)$ for $\mathrm{K} 2-32 \mathrm{e}$, a nominal stellar mass of $0.856 M_{\odot}$, and a semimajor axis of $0.04951 \mathrm{AU}$ (Table 2), we estimate a stellar radial velocity (RV) amplitude of $0.44 \mathrm{~m} \mathrm{~s}^{-1}$, which seems just beyond the reach of modern spectrographs (Dai et al. 2016; Petigura et al. 2017). Even if K2-32 e were made up fully of iron, its mass of then roughly $1.4 M_{\oplus}$ would imply an $\mathrm{RV}$ amplitude of just about $0.6 \mathrm{~m} \mathrm{~s}^{-1}$ and hardly surpass the RV noise (or RV jitter) of several $\mathrm{m} \mathrm{s}^{-1}$ that is typical for solar-type main-sequence stars (Wright 2005).

We also studied whether transit timing variations (TTVs) could be an alternative avenue to determine the planetary masses around K2-32 (Holman \& Murray 2005; Agol et al. 2005). Using our MCMC characterization of the system (Table 1), a mass of 16.5 $M_{\oplus}$ for planet K2-32 b (Petigura et al. 2017), and a nominal Earth mass for K2-32 e, we find that Eq. (8) from Lithwick et al. (2012) predicts a TTV amplitude of $\sim 140 \mathrm{~s}$ or $0.04 \mathrm{~h}$ for K2-32 e. This is probably undetectable in the available Kepler data (see top panel of Fig. 3), but could be tested in a detailed follow-up study.

The near 1:2:5:7 MMR chain of K2-32 e that we report is somewhat reminiscent of other MMR chains, such as the 3:4:6:8 MMR chain observed in the Kepler-223 system (Mills et al. 2016). While the Kepler-223 system exhibits a very precise MMR tuning that has been interpreted as a footprint of planet migration, the fact that K2-32 is substantially non-synchronized with the 1:2:5:7 MMR chain suggests that additional processes have been at work. The origin of these deviations from precise commensurability might be in long-term star-planet tidal interaction that was only overruled by the MMR-creating effects of the protoplanetary disk in the very early stages of the system (Papaloizou 2011; Heller 2018). K2-32 joins the family of planetary systems from the Kepler mission that are just wide of exact commensurability (Lee et al. 2013), although K2-32 represents a particular case with four rather than just two near-resonant planets. Lissauer et al. (2011) defined a new variable

$\zeta_{1}=3\left(\frac{1}{\mathcal{P}-1}-\operatorname{Round}\left(\frac{1}{\mathcal{P}-1}\right)\right)$

as a measure of the difference between an observed first-order MMR period ratio, where $\mathcal{P}=P_{\mathrm{o}} / P_{\mathrm{i}}$ is the orbital period ratio between the outer and the inner planet. These authors found statistically significant deviations of the observed $\zeta_{1}$ distribution from a random period ratio distribution for about 100 transiting exoplanets from Kepler known at the time. Their results showed that for a randomly drawn pair of Kepler planets, $\zeta$ is most likely to range between -0.1 and -0.2 . Taking the median orbital periods from our MCMC sampling for K2-32 e and $\mathrm{b}$ (Table 1), which are very close to the 2:1 MMR resonance $\left(P_{\mathrm{o}} / P_{\mathrm{i}}=2.0676\right)$, we find $\zeta_{1}=-0.19$. Although this 

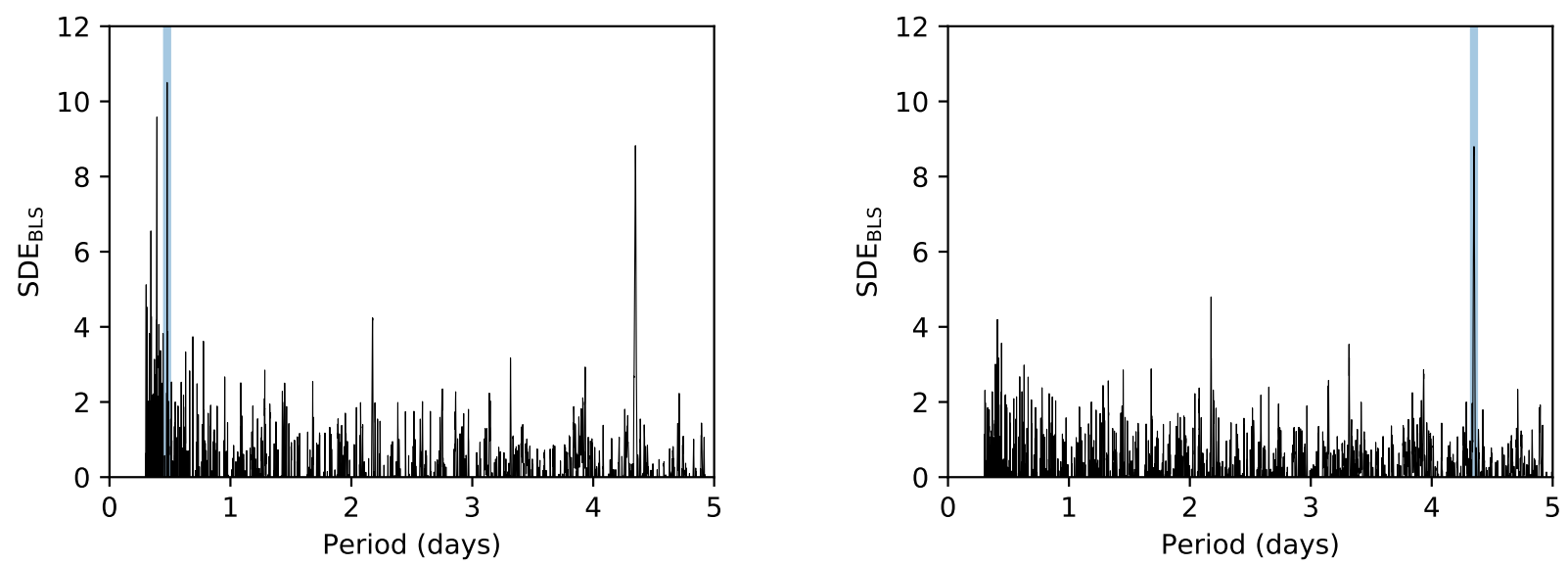

Fig. 6. Left panel: zoom into the bottom left panel of Fig. 5. The major peak near $P=0.4 \mathrm{~d}$ (blue marker) in the $\mathrm{SDE}_{\mathrm{BLS}}$ spectrum is a false positive that is caused by an aliasing effect of the discrete cadence-based duration grid employed by most BLS implementations, here using 10000 trial periods on a linear grid between 0.3 and $5 \mathrm{~d}$. Right panel: same as in the left panel, but now using more than ten times as many trial periods and a nonlinear period grid with the spacing increasing as $P^{1 / 3}$. Although the false-positive peak near $P=0.4 \mathrm{~d}$ has disappeared, the signal of K2-32 e near $P=4.3 \mathrm{~d}$ is still formally a false negative with $\mathrm{SDE}_{\mathrm{BLS}}=8.9$.

result cannot be used as a means of verification for $\mathrm{K} 2-32 \mathrm{e}$, it is in very good agreement with a large sample of exoplanets from Kepler (Lissauer et al. 2011) and therefore serves as an indirect piece of evidence in favor of the planetary nature of $\mathrm{K} 2-32 \mathrm{e}$.

\subsection{Aliasing effects in BLS}

Figure 5 shows the results we obtained with the BLS reference implementation in astropy. Nevertheless, the question arises if the occurrence of a false positive near $P=0.4 \mathrm{~d}$ could be avoided with different grids for the trial periods and trial durations. We therefore varied the resolution and spacing of both the duration and the period grids for BLS to determine whether we could remove this alias peak. The results are illustrated in Fig. 6 and are discussed in the following.

First, the grid of trial transit durations used in BLS must be in multiples of a constant cadence. As an intrinsic property of BLS, any given cadence is tested for one of two test flux values that relate to either the out-of-transit flux or to the in-transit flux (Kovács et al. 2002). As a consequence, when BLS is applied with a period grid of constant spacing, short trial periods may happen to be integer multiples of the trial duration. For example, a trial period of $0.416 \mathrm{~d}$ is sufficiently close to the 20-fold multiple of a $30 \mathrm{~min}$ trial transit duration (a single long-cadence exposure of a $\mathrm{K} 2$ light curve), and a 10-fold multiple of a $60 \mathrm{~min}$ transit duration (worth two cadences), etc. As the transit duration grows with the orbital period to the power of one-third (Eq. (10) in Hippke \& Heller 2019), everything else being equal, aliasing becomes less of an issue for longer trial periods. Nevertheless, aliasing cannot be avoided completely by using an infinitely fine period grid. Instead, it can be avoided by using a nonlinear period grid as employed by TLS.

Second, the grid of trial durations tested with BLS is usually the same for all trial periods, see, for example, the BLS implementation in astropy. Although users can define a maximum trial duration of, for example, $\sim 0.21$ days (or 10 cadences), which is sufficiently long for most long-period planets in $\mathrm{K} 2$, this trial duration is as much as half of an orbital period for the most shortperiod planets that are physically plausible. Again, clustering of data can generate aliases such as those visible near $P=0.3 \mathrm{~d}$ in Fig. 6 (left panel) and eventually lead to false-positive detections. In this example, the most reliable way for us to remove the alias peaks was to use a hyperfine nonlinear grid of $>100000$ trial periods in combination with a duration grid that uses a maximum trial duration shorter than half of the shortest period. Nevertheless, and maybe most important, the signal of K2-32 e near $P=4.3 \mathrm{~d}$ remained a false negative for the BLS search.

In practice, it could be possible to develop more appropriate trial period and trial duration grids for BLS similar to the grid that is implemented in TLS, but this is beyond the scope of this paper, and the disadvantage of the suboptimal detector shape (box versus limb-darkened transit) would remain.

\section{Conclusions}

We determined with high significance $\left(\mathrm{SDE}_{\mathrm{TLS}}=26.1\right)$ a fourth sequence of periodic transits in the light curve of K2-32. Our MCMC simulations suggest that this signal has a period of $4.34882_{-0.00075}^{+0.00069} \mathrm{~d}$ and the transiting planet has a radius of $1.01_{-0.09}^{+0.10} R_{\oplus}$, making it one of the smallest planets found with $\mathrm{K} 2$ so far. Our false-positive vetting of K2-32 e as an individual transiting object yields FPP $=3.1 \times 10^{-3}$. Factoring in the planetary multiplicity around K2-32, we find FPP $<3.1 \times 10^{-4}$. This formally validates $\mathrm{K} 2-32 \mathrm{e}$ as a planet.

This new planet reveals a near 1:2:5:7 MMR chain of now four planets around K2-32. While being very close to this MMR chain, however, the planets are in fact just wide of exact commensurability, thereby joining a growing family of this type of systems from the Kepler mission. We find that the offset from exact commensurability between the new planet and the previously know K2-32 b is in very good agreement with the offsets found in other multiplanet transiting systems, adding more evidence in favor of the planetary nature of K2-32 e. K2-32 also joins the list of $\mathrm{K} 2$ systems with four or more transiting planet candidates, about a dozen of which are known as of today. For now, K2-138 is the only system with five (Christiansen et al. 2018) or potentially even six (Hardegree-Ullman \& Christiansen 2019) transiting planets, all of which are in the super-Earth to sub-Neptune radius regime.

Our discovery confirms that TLS can find sub-Earth-sized planets that have previously been missed with search algorithms 
looking for box-like transit signals such as BLS (Kovács et al. 2002). We verified that there are two reasons why previous searches have missed K2-32 e. First, we used the K2 light curve of K2-32 that was subject to the highly efficient removal of systematic effects with EVEREST (Luger et al. 2016), while previous searches used the light curve detrended with K2SFF that has slightly less favorable noise properties. Second, TLS is intrinsically more efficient in finding shallow transits because the search function is a transit and not a box (Hippke \& Heller 2019). Interestingly, we find that K2-32 e could have been detected in the K2SFF light curve with TLS.

Acknowledgements. The authors thank the referee for a helpful report. This research has made use of the NASA Exoplanet Archive, which is operated by the California Institute of Technology, under contract with the National Aeronautics and Space Administration under the Exoplanet Exploration Program. This work made use of NASA's ADS Bibliographic Services. R.H. is supported by the German space agency (Deutsches Zentrum für Luft- und Raumfahrt) under PLATO Data Center grant 50001501. K.R. is a member of the International Max Planck Research School for Solar System Science at the University of Göttingen. K.R. performed the MCMC analysis of the light curve.

\section{References}

Agol, E., Steffen, J., Sari, R., \& Clarkson, W. 2005, MNRAS, 359, 567

Batalha, N. M., Rowe, J. F., Bryson, S. T., et al. 2013, ApJS, 204, 24

Borucki, W. J., Koch, D., Basri, G., et al. 2010, Science, 327, 977

Christiansen, J. L., Crossfield, I. J. M., Barentsen, G., et al. 2018, AJ, 155, 57

Crossfield, I. J. M., Ciardi, D. R., Petigura, E. A., et al. 2016, ApJS, 226, 7

Crossfield, I. J. M., Guerrero, N., David, T., et al. 2018, ApJS, 239, 5

Cutri, R. M., Skrutskie, M. F., van Dyk, S., et al. 2003, VizieR Online Data Catalog: II/246

Dai, F., Winn, J. N., Albrecht, S., et al. 2016, ApJ, 823, 115

Foreman-Mackey, D., Hogg, D., Lang, D., \& Goodman, J. 2013, PASP, 125, 306
Hardegree-Ullman, K., \& Christiansen, J. 2019, American Astronomical Society Meeting, 233, 164.07

Heller, R. 2018, A\&A, submitted [arXiv:1806.06601]

Hippke, M., \& Heller, R. 2019, A\&A, 623, A39

Holman, M. J., \& Murray, N. W. 2005, Science, 307, 1288

Howell, S. B., Sobeck, C., Haas, M., et al. 2014, PASP, 126, 398

Huber, D., Bryson, S. T., Haas, M. R., et al. 2016, ApJS, 224, 2

Kepler, J. 1619, Harmonices mundi libri V (Austria: Linz)

Kipping, D. M. 2013, MNRAS, 435, 2152

Kovács, G., Zucker, S., \& Mazeh, T. 2002, A\&A, 391, 369

Lee, M. H., Fabrycky, D., \& Lin, D. N. C. 2013, ApJ, 774, 52

Lissauer, J. J., Ragozzine, D., Fabrycky, D. C., et al. 2011, ApJS, 197, 8

Lissauer, J. J., Marcy, G. W., Rowe, J. F., et al. 2012, ApJ, 750, 112

Lithwick, Y., Xie, J., \& Wu, Y. 2012, ApJ, 761, 122

Livingston, J. H., Endl, M., Dai, F., et al. 2018a, AJ, 156, 78

Livingston, J. H., Crossfield, I. J. M., Petigura, E. A., et al. 2018b, AJ, 156, 277

Luger, R., Agol, E., Kruse, E., et al. 2016, AJ, 152, 100

Luger, R., Kruse, E., Foreman-Mackey, D., Agol, E., \& Saunders, N. 2018, AJ, 156,99

Mandel, K., \& Agol, E. 2002, ApJ, 580, L171

Manduca, A., Bell, R. A., \& Gustafsson, B. 1977, A\&A, 61, 809

Mayo, A. W., Vanderburg, A., Latham, D. W., et al. 2018, AJ, 155, 136

Mills, S. M., Fabrycky, D. C., Migaszewski, C., et al. 2016, Nature, 533, 509

Morton, T. D. 2012, ApJ, 761, 6

Morton, T. D. 2015, VESPA: False positive probabilities calculator, Astrophysics Source Code Library [record ascl : 1503.011]

Ofir, A. 2014, A\&A, 561, A138

Papaloizou, J. 2011, Celest. Mech. Dyn. Astron., 111, 83

Petigura, E. A., Howard, A. W., \& Marcy, G. W. 2013a, PNAS, 110, 19273

Petigura, E. A., Marcy, G. W., \& Howard, A. W. 2013b, ApJ, 770, 69

Petigura, E. A., Sinukoff, E., Lopez, E. D., et al. 2017, AJ, 153, 142

Petigura, E. A., Crossfield, I. J. M., Isaacson, H., et al. 2018, AJ, 155, 21

Sinukoff, E., Howard, A. W., Petigura, E. A., et al. 2016, ApJ, 827, 78

van Sluijs, L., \& Van Eylen V. 2018, MNRAS, 474, 4603

Vanderburg, A., \& Johnson, J. A. 2014, PASP, 126, 948

Vanderburg, A., Latham, D. W., Buchhave, L. A., et al. 2016, ApJS, 222, 14

Wright, J. T. 2005, PASP, 117, 657

Yu, L., Crossfield, I. J. M., Schlieder, J. E., et al. 2018, AJ, 156, 22 